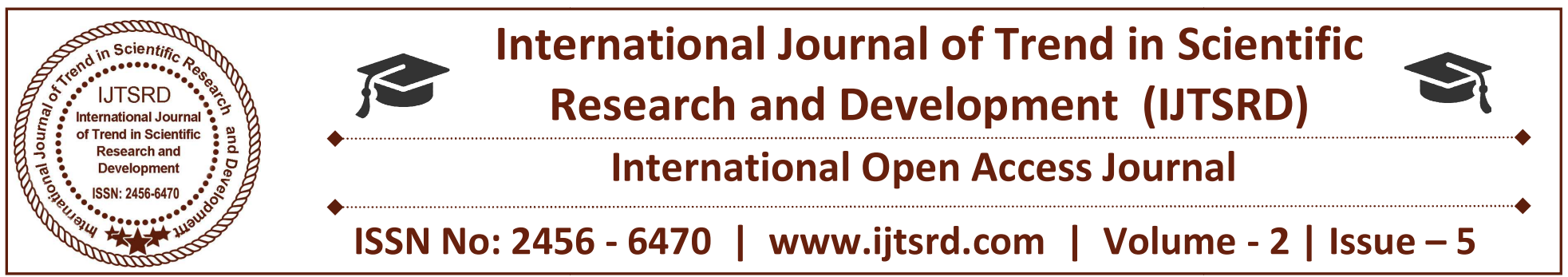

\title{
A Delay Differential Equation Model of Hepatitis B Virus (HBV) Infection and Immune Control
}

\author{
Titus Ifeanyi Chinebu ${ }^{1}$, Edmund Onwubiko Ezennorom ${ }^{1}$, Godwin C. E. Mbah ${ }^{2}$ \\ ${ }^{1,2}$ Department of Computer Science, Madonna University Nigeria, Elele, Nigeria \\ ${ }^{3}$ Department of Mathematics, University Of Nigeria, Nsukka, Nigeria
}

\begin{abstract}
We investigated the intracellular delay effect on the stability of the endemically infected steady state by analyzing a nonlinear ordinary differential equation model of hepatitis $B$ virus (HBV) infection that considers the interaction betweena replicating virus, hepatocytes and the cytotoxic T/ymphocytes (CTL). We gave a criterion to ensure that the infected steady state is asymptotically stable for all delays. A critical delay below which the CTL (immune control mechanism) can be significantly helpful in controlling the HBV infection even when the basic reproduction number is high is allowed in the analysis.
\end{abstract}

KEYWORD: Delay differential Equation, Critical Delay, Hepatitis B Virus, immune system

\section{INTRODUCTION}

Hepatitis B virus (HBV) infection is a major health problem worldwide and despite the presence of hepatitis $\mathrm{B}$ vaccine, new $\mathrm{HBV}$ infections remain common. HBV is a hepatotropic double stranded DNA virus belonging to the hepadnaviridae family. One of the five hepatitis viruses, HBV causes acute and chronic hepatitis in humans. Acute HBV infection causes only mild symptoms. Majority of infected adults successfully clear the virus and acquires lifelong immunity. It takes about 1 to 6 months from the time of infection for the disease to manifest itself and early symptoms include nausea and vomiting, loss of appetite, fatigue, with dark urine and light stools follows. Only about one percent of patients infected with hepatitis B virus die due to liver damage in their early stage (Poynard, 2002). The risk of becoming chronically infected depends on the age at the time of infection. More than 90 percent of new born, 50 percent of children, 5 percent of adults infected with
HBV develop chronic hepatitis (WHO, 2014). Those who are unable to produce an effective immune response allow the virus to replicate for long periods in their liver causing chronic hepatitis B virus, (HBV) infection, cirrhosis of liver and hepatocellular carcinoma (HCC) (Yen, 2002). Chronic HBV infection is shown to be $0.8 \%$ in pregnant women with the risk of vertical (mother to child) transmission (Trehanpati et al, 2013). In neonates, HBV infection has a linkage with strong presence of Tregs ( $\mathrm{T}$ regulatory cells) and defective $\mathrm{CD}^{+} \mathrm{T}$ cells pool to produce interferon $(I F N)-\gamma$. Associated with decreased $\mathrm{CD}^{+} \mathrm{T}$ cell dysfunction is $\mathrm{T}$ cell receptor $(T C R \zeta)$ chain. Due to persistent intrauterine exposure of the viral antigens early in embryonic development there could be $T C R \zeta$ leading to immune tolerance to $\mathrm{HBV}$ antigens in the newborns positive for hepatitis B surface antigen ( $\mathrm{HBsAg}+\mathrm{ve})$. Finally $\mathrm{T}$ cell tolerance to $\mathrm{HBV}$ antigen due to $\mathrm{HBV}$ infection may probably develop to chronicity in the newborn. To restore acquired immunity and better production of HBV specific antibodies, vaccination may be necessary (Trehanpati et al, 2013).

Two disease states, not necessarily static, are possible after seroclearance of $\mathrm{HBeAg}$ and they are inactive carrier (IC) state and chronic hepatitis B (CHB) state (Pita et al, 2014). The duration of clearance (reactive) phase last from months to years. In the inactive carrier (IC) state, seroconversion of hepatitis $\mathrm{B}$ e antigen (HBeAg) to hepatitis B e antibody (HBeAb) occurs, HBV DNA becomes non detectable or at low level and ALT is usually normal, reflecting very low or no replication of $\mathrm{HBV}$ and mild or no hepatic injury (European association for the study of liver (EASL), 
2012). The inactive carrier state may last for years or even lifetime. Patients in this state can have spontaneous resolution of hepatitis $\mathrm{B}$ and develop HBsAg, but a portion of them may undergo spontaneous or immunosuppressant - induced reactivation of chronic hepatitis $B$. This bring in the second state which is chronic hepatitis B and is usually associated with elevated ALT, high level of DNA, moderate to severe liver histological activity, and with or without $\mathrm{HBeAg}$ seroconversion (Pan and Zhang, 2005). Differentiating between inactive carrier (IC) and chronic hepatitis B (CHB) status is very important in clinical practice, because it has an implication in the follow-up, management and prognosis (EASL, 2012).

The HBV virion genome is circular and approximately $3.2 \mathrm{~kb}$ in size, consisting of DNA that is mostly double stranded. It has compact organization, with four overlapping reading frames running in one direction and no non coding regions. The minus strand is unit length and has a protein covalently attached to the $5^{\prime}$ end. Thus, neither DNA strand, is closed and circularity is maintained by cohesive ends (Strauss, 2002). The four overlapping open reading frames (ORFs) in the genome are responsible for the transcription and expression of seven different hepatitis B proteins. The translation of these proteins is through the use of multiple in-frame start codons. The HBV genome also contains parts that regulate transcription, determine the site of polyadenylation and a specific nucleocapsid. The genomic arrangement of the hepatitis B virus family makes it unique among viruses. The unusually packaged may indicate that the method of replication employed by $\mathrm{HBV}$ is not of conserved DNA replication (Graces, HBVP, 2003).

Since HBV is a noncytopathic virus (Ilan, 2002), that is cells infected by HBV will not be killed by virus directly, cellular function and life span of HBV infected hepatocytes are almost the same as that of the uninfected cells in vitro (Kangxian, 2006). The death rate of noncytopathic virus infected cells in the absence of immunity equals that of uninfected target cells (Woderz, 2005). The lifespan of HBV infected cells varies greatly in vivo which is mainly due to the strength of the anti HBV CTL response (Nowak and Robert, 2000). CTL will not only kill but cure the infected hepatocytes by nonlytic effector mechanism (Bartolett a and Ferrari, 2003; and Guidotti, 2003). In a study by Yang et al (2009) it was observed that, induction of a $C D 8^{+} \mathrm{T}$ cell response depends on the presence of $C D 4^{+} \mathrm{T}$ cells. That each of these effectors, $C D 4^{+}$and $C D 8^{+} \mathrm{T}$ cells, Nk cells, fas, IFN - gamma (IFN - $\gamma$ ), IFN - alpha/beta receptor (IFN $\alpha / \beta-\mathrm{R}$ ), and IFN receptor 1 (INF R1), was required to eliminate the transcriptional template from the liver. These results are consistent with a model in which $C D 4^{+} \mathrm{T}$ cells serve as master reguletors of the adaptive immune response to $\mathrm{HBV} ; \mathrm{CDB}^{+} \mathrm{T}$ cells are the key cellular effectors mediating HBV clearance from the liver, apparently by a fas - dependent perforin independent process in which NK cells, IFN $-\gamma$, INF R1 and IFN $-\alpha / \beta-\mathrm{R}$ play supportive roles (Yang et al, 2009). Immediately after infection, innate immunity limits the spread of the pathogen and initiate efficient development of an adaptive immune response. Innate host responses during the early phase of viral infection are mainly characterized by the production of type 1 interferon (IFN) $-\alpha / \beta$ cytokines and the activation of natural killer (NK) cells. Production of type 1 IFNs can be triggered directly by virus replication through cellular mechanisms that detect the presence of viral RNA or DNA (Alexopoulou et al, 2001; Lund et al, 2003; Heil et al, 2004), while NK cells are activated by the recognition of stress - induced molecules and /or the modulation of the quantity of major histocompatibility complex (MHC) - class 1 molecules on the surface of infected cells (Moretta et al, 2005).

\section{RELATED WORKS}

$\mathrm{Wu}$ and $\mathrm{Su}$ (2014) considered a model with intracellular delay and nonlinear infection rate of saturated functional response. By constructing suitable Lyapunov functions and using LaSalle invariance principle, the proved that the infection free equilibrium is globally asymptotiacally stable when $R_{0} \leq 1$. And if $R_{0}>1$, there exists an infection equilibrium and they get its global asymptotic stability by constructing Lyapunov functional. Wang and Tian (2013) developed and investigated a model that studied the global stability for a delayed HBV infection with CTL immune response. They showed that the global dynamics is determined by the basic reproduction number $R_{0}$ and CTL immune response reproduction number $R_{1}$.

Huang et al (2010) studied the global properties of a class of delay differential equations model for virus infection with nonlinear transmissions. Their model gives a more complex and general infection process, a general nonlinear contact rate between target cells and 
viruses and the removal rate of infected cells are considered, and two constant delays are incorporated into their model. These incorporated delays describes (i) the time needed for a newly infected cells to start producing viruses and (ii) the time needed for a newly produced virus to become infectious (mature). Wang et al (2007) developed and analyzed the HBV infection in a diffusion model confined to a finit domain, induced by intracellular time delay between infection of a cell and production of a new viral particle. Rezounenko (2017) investigated a class of reaction diffusion virus dynamics model with intracellular state dependent delay and a nonlinear infection rate functional response.

\section{Formulation of the Model Equations}

\subsection{The Existing Model}

We start our model formulation by introducing the model by Chinebu et al (2017) and finally incorporate the delay parameter to obtain the delay model.

$$
\begin{gathered}
\frac{d x}{d t}=\psi-a_{1} x-\frac{\beta a_{3} x y}{\mu}+b_{1} y z \\
\frac{d y}{d t}=\zeta y+\frac{\beta a_{3} x y}{\mu}-a_{1} y-\left(b_{1}+b_{2}\right) y z \\
\frac{d z}{d t}=\gamma+a_{4} y z-b_{4} z .
\end{gathered}
$$

Equation (3.1) contains three variables, that is, uninfected hepatocytes $(x)$, infected hepatocytes $(y)$, and CTL response( $z)$. Uninfected, susceptible hepatocyte are assumed to be produced at a constant level, $\psi$, the natural death rate of both infected and uninfected hepatocytes are assumed to be the constant $a_{1} \cdot \frac{\beta a_{3} x y}{\mu}$ is assumed to be the level at which uninfected hepatocytes are being infected due to interaction with infected hepatocytes and $\zeta y$ is the level at which infected hepatocyte proliferate. The elimination of virus is through the response of the cytotoxic $\mathrm{T}$ lymphocytes (CTL) which can be activated in two different pathways, either by killing the infected hepatocytes or by clearing the virus from within the hepatocyte without killing it. Infected hepatocytes are assumed to be killed by the CTL response at a rate $b_{2} y z$ and be cleared from within by the CTL response at a rate $b_{1} y z . a_{3} y$ is the production level of free virus from infected hepatocytes and the free virus particles are removed at a rate $\mu$.Two terms, $\gamma$ and $a_{4} y z$ were used to describe
CTL rapid growth and reproduction of new cells (proliferation), where $\gamma$ represents antigen independent proliferation (primary immune response) and $a_{4} y z$ represents antigen dependent proliferation (secondary immune response). $\gamma$ is small, and is the response to the first exposure to antigen which is mediated by lymphocytes, called naïve lymphocytes that are seeing antigen for the first time and they are "immunologically inexperienced". We also assume that CTL decay at constant rate $b_{4}$ (Chinebu et al, 2017).

\subsection{Delay Differential Equation}

A delay differential equation is a type of differential equation whereby the derivative of the unknown function at a certain time is given in terms of the values of the function at previous time and is also known as time delay system. Notice that we did not incorporate any time delay in HBV proliferation in the model presented in equation 3.1. Between the infection of a cell and the viral particles emission, virus production may lag by an intracellular time delay $\tau$. The delay or lag represents incubation period, thereby accounting only for the time required for the production of new virus particles. However, Rotich and Lag at (2014), shows that when the drug efficacy is less than perfect, the intracellular delay will be of crucial importance because the delay depends on the live span of the liver cell, that is to say that the maximum length of time that the cell will survive after infection is considered. The incorporation of intracellular delay is assumed to give a clearer view in the interpretation of the analytical result and this has important implications on therapeutic options and drug development.

Gourley et al (2008), developed a delay differential equations on HBV infection which explicitly incorporate a time delay in virus production. For their model, the existence and the component values of the endemic steady state are explicitly dependent on the time delay. They further stated that exposed cells begin shedding virions after $\tau$ unit of time, representing the time required to construct, transcribe and translate the viral genome. The episomal viral genome, construct and then release mature virions. There work extended the ability of models to describe the relevant biological process and addressed the implication of ignoring the intracellular delays that are part of the viral life cycle. However, they did not take into cognizance that cytotoxic $\mathrm{T}$ lymphocytes 
play an important role in the immunological mechanism as will be seen in our later discussion.

Consider a system that can be in either one of two states Q (infection of a cell) and R (viral particle emission). Suppose that at timet $=0$, the system is in state Q. suppose that when event $p$ takes place, the system switches to state R. Modifying the model given by (3.1), we have a new model which assumes a delay $\tau$, from the time of infection until the production of new virus particles. We define a translation operator in order to describe the delay in mathematical terms.

For a real - value function $p$ and for $\tau \geq 0$, we define the translation operator $\mathrm{T}_{\tau}$ by

$\left(\mathrm{T}_{\tau} p\right)(t)=p(t-\tau)$, with time delay $t \geq 0$ and $p$ $\in \mathbb{R}^{n}$.

Since the virus must have contact with the liver cells before they will be infected, we let

$$
x_{1}=\mathrm{T}_{\tau} x, \quad y_{1}=\mathrm{T}_{\tau} y, \quad z_{1}=\mathrm{T}_{\tau} z
$$

for notional convenience and this gives the new system with delay as

$$
\begin{gathered}
\frac{d x}{d t}=\psi-a_{1} x-\frac{\beta a_{3} x y}{\mu}+b_{1} y z \\
\frac{d y}{d t}=\zeta y+\frac{\beta a_{3} x_{1} y_{1}}{\mu}-a_{1} y-\left(b_{1}+b_{2}\right) y z \\
\frac{d z}{d t}=\gamma+a_{4} y z-b_{4} z .
\end{gathered}
$$

$\frac{\beta a_{3} x_{1} y_{1}}{\mu}$, indicates a finite time lag between infection of a hepatocyte and production of new virus particles in the equation that determine the rate of $y$ and this describes the dynamical variable $y$ at time $t$ that depends on the term evaluated at earlier time $t-\tau$.

Since there are three, possible steady state in the previous section and these states are too complex to analyze, we therefore, discuss only the linear ability of the most concerned state - uninfected state. System (3.2) is a special case of the general system:

$$
\begin{array}{r}
\frac{d x}{d t}=F_{1}\left(x, y, z, x_{1}, y_{1}, z_{1}\right) \\
\frac{d y}{d t}=F_{2}\left(x, y, z, x_{1}, y_{1}, z_{1}\right) \\
\frac{d z}{d t}=F_{3}\left(x, y, z, x_{1}, y_{1}, z_{1}\right)
\end{array}
$$

This simply shows that the steady states of system (3.2) and (3.1) are the same. Considering a neighborhood close to the steady state solution and letting

Where functions $F_{1}, F_{2}$ and $F_{3}$ depends on $x, y, z$ and their delays. Therefore, $F_{1}, F_{2}$ and $F_{3}$ can be identified as follows:

$$
\begin{gathered}
F_{1}\left(x, y, z, x_{1}, y_{1}, z_{1}\right)=\psi-a_{1} x-\frac{\beta a_{3} x y}{\mu}+b_{1} y z \\
F_{2}\left(x, y, z, x_{1}, y_{1}, z_{1}\right) \\
=\zeta y+\frac{\beta a_{3} x_{1} y_{1}}{\mu}-a_{1} y \\
-\left(b_{1}+b_{2}\right) y z
\end{gathered}
$$

We then use define the steady state for the delay system having that a point $(\hat{x}, \hat{y}, \hat{z})$ is called a steady state of the system (3.3) if it is a constant solution of the equations.

$$
\begin{gathered}
F_{1}\left(\hat{x}, \hat{y}, \hat{z}, \hat{x}_{1}, \hat{y}_{1}, \hat{z}_{1}\right) \\
F_{2}\left(\hat{x}, \hat{y}, \hat{z}, \hat{x}_{1}, \hat{y}_{1}, \hat{z}_{1}\right) \\
F_{3}\left(\hat{x}, \hat{y}, \hat{z}, \hat{x}_{1}, \hat{y}_{1}, \hat{z}_{1}\right)
\end{gathered}
$$
then the steady state of system (3.2) is the same as the steady state of system (3.1).

Proof: From equation (3.4), we have that

$$
\begin{aligned}
& F_{1}\left(\hat{x}, \hat{y}, \hat{z}, \hat{x}_{1}, \hat{y}_{1}, \hat{z}_{1}\right)=f_{1}(\hat{x}, \hat{y}, \hat{z}) \\
& F_{2}\left(\hat{x}, \hat{y}, \hat{z}, \hat{x}_{1}, \hat{y}_{1}, \hat{z}_{1}\right)=f_{2}(\hat{x}, \hat{y}, \hat{z}) \\
& F_{3}\left(\hat{x}, \hat{y}, \hat{z}, \hat{x}_{1}, \hat{y}_{1}, \hat{z}_{1}\right)=f_{3}(\hat{x}, \hat{y}, \hat{z})
\end{aligned}
$$

$$
\begin{array}{ccc}
x=\hat{x}+X, & y=\hat{y}+Y, & z=\hat{z}+Z, \\
x_{1}=\hat{x}+X_{1}, & y_{1}=\hat{y}+Y_{1}, & z_{1}=\hat{z}+Z_{1}
\end{array}
$$

after which we expand $F_{1}, F_{2}$ and $F_{3}$ in the Taylor series about the point $(\hat{x}, \hat{y}, \hat{z})$ and retain only the linear terms, to obtain

$$
\begin{gathered}
\frac{d X}{d t}=\left[-a_{1}-\frac{\beta a_{3} \hat{y}}{\mu}\right] X+\left[-\frac{\beta a_{3} \hat{x}}{\mu}+b_{1} \hat{z}\right] Y+\left[b_{1} \hat{y}\right] Z \\
\begin{aligned}
\frac{d Y}{d t}=\left[\frac{\beta a_{3} \hat{y}}{\mu}\right] & X_{1}+\left[\frac{\beta a_{3} \hat{x}}{\mu}\right] Y_{1} \\
+ & {\left[\zeta-a_{1}-\left(b_{1}+b_{2}\right) \hat{z}\right] Y } \\
- & {\left[\left(b_{1}+b_{2}\right) \hat{y}\right] Z }
\end{aligned}
\end{gathered}
$$

$$
\frac{d Z}{d t}=\left[a_{4} \hat{z}\right] Y+\left[a_{4} \hat{y}-b_{4}\right] Z
$$

Proposition1: If $a_{1}, a_{3}, b_{1}, b_{2}, b_{4}, \psi, \zeta, \gamma, \beta, \mu>0$, 
To linearize (3.6) we let

$$
\left[\begin{array}{l}
\frac{d X}{d t} \\
\frac{d Y}{d t} \\
\frac{d Z}{d t}
\end{array}\right]=\left[\begin{array}{l}
x(t) \\
y(t) \\
z(t)
\end{array}\right]
$$

Let

$$
\left[\begin{array}{l}
x \\
y \\
z
\end{array}\right]=H \text { and }\left[\begin{array}{l}
x \\
y \\
z
\end{array}\right](t)=H(t)
$$

But, in biological population (Malthusian growth model), the major underlying assumption is that the rate of change of population is proportional to the population itself.

Thus we let

$$
\frac{d H}{d t}=\lambda H
$$

where the parameter $\lambda$ is known as the natural rate of increase and is a positive constant. We now solve the differential equation using the method of separation of variables to obtain

$$
\begin{gathered}
\frac{d H}{H}=\lambda d t . \\
\int \frac{d H}{H}=\int \lambda d t
\end{gathered}
$$$$
\ln H=\lambda t+c
$$

We take exponential of both sides to get

$$
\begin{gathered}
e^{\ln H}=e^{\lambda t+c} \\
\Rightarrow H(t)=e^{\lambda t} \cdot e^{c}
\end{gathered}
$$

If we let $e^{c}=H_{0}$ we have

$$
H(t)=H_{0} e^{\lambda t} .
$$

This predicts exponential population growth as $t$ increases. However, due to the time lag between the infection of a hepatocyte and viral particle emission from the hepatocyte, it may be more realistic to assume that the instantaneous rate of change of population growth is actually dependent upon the population at some fixed amount of time $\tau$ in the past (Cain and Raynolds, 2006). Observe that the rate of change of $\mathrm{H}$ at time $t$ is affected by the value of $\mathrm{H}$ at time $t-\tau$. Therefore we have

$$
\begin{gathered}
H(t)=H_{0} e^{\lambda(t-\tau)} \\
\Rightarrow H(t)=H_{0} e^{\lambda t} \cdot e^{-\lambda \tau}
\end{gathered}
$$

At $t=0$ which is assumed to be the time of infection of a hepatocyte, we have

$$
H(t)=H_{0} e^{-\lambda \tau}
$$

where $H_{0}$ is a constant vector (Mac Donald, 1970) and we have

$$
\left[\begin{array}{l}
x_{1} \\
y_{1} \\
z_{1}
\end{array}\right]=e^{-\lambda t}\left[\begin{array}{l}
x \\
y \\
z
\end{array}\right]
$$

which transforms system (4.22) into a linear system as stated below

$$
\left[\begin{array}{l}
\frac{d X}{d t} \\
\frac{d Y}{d t} \\
\frac{d Z}{d t}
\end{array}\right]=\left[\begin{array}{ccc}
-\left(a_{1}+\frac{\beta a_{3} \hat{y}}{\mu}\right) & -\left(\frac{\beta a_{3} \hat{x}}{\mu}-b_{1} \hat{z}\right) & b_{1} \hat{y} \\
\frac{\beta a_{3} \hat{y} e^{-\lambda t}}{\mu} & \zeta+\frac{\beta a_{3} \hat{x} e^{-\lambda t}}{\mu}-a_{1}-\left(b_{1}+b_{2}\right) \hat{z} & -\left(b_{1}+b_{2}\right) \hat{y} \\
0 & a_{4} \hat{z} & a_{4} \hat{y}-b_{4}
\end{array}\right]\left[\begin{array}{l}
X \\
Y \\
Z
\end{array}\right] .
$$

Then, we arrive at the matrix

$$
H=\left[\begin{array}{ccc}
-\left(a_{1}+\frac{\beta a_{3} \hat{y}}{\mu}\right) & -\left(\frac{\beta a_{3} \hat{x}}{\mu}-b_{1} \hat{z}\right) & b_{1} \hat{y} \\
\frac{\beta a_{3} \hat{y} e^{-\lambda t}}{\mu} & \zeta+\frac{\beta a_{3} \hat{x} e^{-\lambda t}}{\mu}-a_{1}-\left(b_{1}+b_{2}\right) \hat{z} & -\left(b_{1}+b_{2}\right) \hat{y} \\
0 & a_{4} \hat{z} & a_{4} \hat{y}-b_{4}
\end{array}\right]
$$

Equation (3.7) is the coefficient matrix of the linear system having $\lambda$ as the eigenvalue of the matrix $H$. At this point, we investigate the stability of the delay system in terms of the eigenvalues of the matrix $H$. 
Delay differential system requires information on the entire interval $\left[t_{0}-\tau, t_{0}\right]$. To know the rate of change at $t_{0}$, we need $x\left(t_{0}\right), y\left(t_{0}\right), z\left(t_{0}\right), x\left(t_{0}-\tau\right), y\left(t_{0}-\tau\right)$ and $z\left(t_{0}-\tau\right)$. According to Burton (1985), it is well known that the solution to $s^{\prime}(t)=s(t)^{2}$ diverge to infinity in finite time. Solutions to the delay differential equation $s^{\prime}(t)=s(t-\tau)^{2}$, however, are continuable for all time if $\tau$ is positive for $t$. Also,Halanay (1966), states that if $\left(x^{*}, y^{*}, z^{*}\right)$ is the steady state solution of system (3.3), we say that $\left(x^{*}, y^{*}, z^{*}\right)$ is stable if for every $\varepsilon>0$ there is a $\delta>0$ such that, for every solution $(x, y, z)$, if

$$
\left[x\left(t_{0}\right)-x^{*}\right]^{2}+\left[y\left(t_{0}\right)-y^{*}\right]^{2}+\left[z\left(t_{0}\right)-z^{*}\right]^{2}<\delta^{2}
$$

For some $t_{0} \in\left[t_{1}-\tau, t_{1}\right]$, then

$$
\left[x\left(t_{0}\right)-x^{*}\right]^{2}+\left[y\left(t_{0}\right)-y^{*}\right]^{2}+\left[z\left(t_{0}\right)-z^{*}\right]^{2}<\varepsilon^{2} \forall t>t_{1} .
$$

If, $\left(x^{*}, y^{*}, z^{*}\right)$ is not stable, we say that it is unstable. If $\left(x^{*}, y^{*}, z^{*}\right)$ is stable and if $\delta$ can be chosen so that (3.8) implies that $(x(t), y(t), z(t)) \rightarrow\left(x^{*}, y^{*}, z^{*}\right)$ as $t \rightarrow \infty$, we say that $\left(x^{*}, y^{*}, z^{*}\right)$ is asymptotically stable. From Halanay (1966), we remark that the steady state $(\hat{x}, \hat{y}, \hat{z})$ is stable if no eigenvalue of $\mathrm{H}$ has positive real part. We compute the characteristic equation by seeking for exponential solution of the delay differential system to arrive at a transcendental equation of the form

$$
d_{j}(\lambda)+k_{j}(\lambda) e^{-\lambda \tau}=0,
$$

Where $\tau$ is the length of discrete delay added, $d$ and $k$ are polynomial in $\lambda$ and $j=1,2,3$.

Proposition2: Suppose

$$
a_{1}, a_{3}, b_{1}, b_{2}, b_{4}, \gamma, \zeta, \beta, \mu, \psi>0
$$

1. If $a_{1}+\frac{\left(b_{1}+b_{2}\right) \gamma}{b_{4}}>\zeta+\frac{\beta a_{3} \psi}{a_{1} \mu}$, then the steady state $\left(\psi / a_{1}, 0, \gamma / b_{4}\right)$ of the system (18) is asymptomatically stable for $\tau \geq 0$.

2. If $a_{1}+\frac{\left(b_{1}+b_{2}\right) \gamma}{b_{4}}<\zeta+\frac{\beta a_{3} \psi}{a_{1} \mu}$ then the steady state $\left(\psi / a_{1}, 0, \gamma / b_{4}\right)$ of the system (3.2) is unstable for $\tau \geq 0$.

\section{Proof:}

At steady state, the characteristic equation of the delay differential equation will have the form

$U_{2}(\lambda)=\lambda^{3}+\left(d_{1}+k_{1} e^{-\lambda \tau}\right) \lambda^{2}+\left(d_{2}+k_{2} e^{-\lambda \tau}\right) \lambda+\left(d_{3}+k_{3} e^{-\lambda \tau}\right)=0$

which can be written as

$$
\begin{gathered}
U_{2}(\lambda, \tau) \equiv \lambda^{3}+d_{1} \lambda^{2}+d_{2} \lambda+d_{3}+\left(k_{1} \lambda^{2}+k_{2} \lambda+k_{3}\right) e^{-\tau}=0 \\
\left|\begin{array}{ccc}
-\left(a_{1}+\frac{\beta a_{3} \hat{y}}{\mu}\right)-\lambda & -\left(\frac{\beta a_{3} \hat{x}}{\mu}-b_{1} \hat{z}\right) & b_{1} \hat{y} \\
\frac{\beta a_{3} \hat{y} e^{-\lambda t}}{\mu} & \zeta+\frac{\beta a_{3} \hat{x} e^{-\lambda t}}{\mu}-a_{1}-\left(b_{1}+b_{2}\right) \hat{z}-\lambda & -\left(b_{1}+b_{2}\right) \hat{y} \\
0 & a_{4} \hat{z} & a_{4} \hat{y}-b_{4}-\lambda
\end{array}\right|=0 .
\end{gathered}
$$

Since the steady state about which we have linearized is stable in the absence of delay (Chinebu et al, 2017), that is when $\tau=0$,which simply implies that there is no delay and that all the roots of the polynomial have non positive real parts, therefore, proposition 2 is proved as in (Chinebu et al 2017). We further consider the 
situation where $\tau>0$ and therefore substitute the steady state $\left(\psi / a_{1}, 0, \gamma / b_{4}\right)$ into system (3.7), after which we obtain the characteristic equation as;

$$
\begin{aligned}
& \left|\begin{array}{ccc}
-a_{1}-\lambda & -\frac{\beta a_{3} \psi}{a_{1} \mu}+\frac{b_{1} \gamma}{b_{4}} & 0 \\
0 & \zeta+\frac{\beta a_{3} \psi e^{-\lambda t}}{a_{1} \mu}-a_{1}-\left(b_{1}+b_{2}\right) \frac{\gamma}{b_{4}}-\lambda & 0 \\
0 & \frac{a_{4} \gamma}{b_{4}} & -b_{4}-\lambda
\end{array}\right|=0 \\
& \left(\lambda+a_{1}\right)\left(\lambda+a_{1}+\frac{\left(b_{1}+b_{2}\right) \gamma}{b_{4}}-\zeta-\frac{\beta a_{3} \psi e^{-\tau}}{a_{1} \mu}\right)\left(\lambda+b_{4}\right)=0 .
\end{aligned}
$$

Therefore, matrix $H$ has three eigenvalues, at steady state $\left(\psi / a_{1}, 0, \gamma / b_{4}\right)$ and they are $\lambda_{1}=-a_{1}<0, \lambda_{2}=$ $-b_{4}<0$, and $\lambda_{3}$ satisfying the equation

$$
\lambda_{3}+a_{1}+\frac{\left(b_{1}+b_{2}\right) \gamma}{b_{4}}-\zeta-\frac{\beta a_{3} \psi e^{-\tau \lambda}}{a_{1} \mu}<0
$$

At this point, we introduce a function $v(t)$ to enable us solve for the position of $\lambda_{3}$

$$
v(t)=t+a_{1}+\frac{\left(b_{1}+b_{2}\right) \gamma}{b_{4}}-\zeta-\frac{\beta a_{3} \psi e^{-\tau t}}{a_{1} \mu}, t \in \mathbb{R}
$$

and this enable us to find the location of the eigenvalue $\lambda_{3}$. By differentiating $v(t)$ with respect to $t$, yields

$$
v^{\prime}(t)=1+\frac{\beta a_{3} \psi \tau e^{-\tau t}}{a_{1} \mu}
$$

which is always positive, we also observe that

$$
\lim _{t \rightarrow-\infty} v(t)=-\infty, \quad \lim _{t \rightarrow \infty} v(t)=\infty
$$

This simply shows that the function $v$ has a unique zero. That is

$$
v(0)=a_{1}+\frac{\left(b_{1}+b_{2}\right) \gamma}{b_{4}}-\zeta-\frac{\beta a_{3} \psi}{a_{1} \mu}
$$

From here, we observe that if $a_{1}+\frac{\left(b_{1}+b_{2}\right) \gamma}{b_{4}}>\zeta+\frac{\beta a_{3} \psi}{a_{1} \mu}$, we conclude that $v(0)>0$ and thus, $\lambda_{3}<0$ and this implies that the steady state $\left(\psi / a_{1}, 0, \gamma / b_{4}\right)$ is asymptotically stable for all $\tau>0$. If $a_{1}+\frac{\left(b_{1}+b_{2}\right) \gamma}{b_{4}}<\zeta+\frac{\beta a_{3} \psi}{a_{1} \mu}$, we then conclude that $v(0)<0$ and therefore $\lambda_{3}>0$ which shows that the steady state $\left(\psi / a_{1}, 0, \gamma / b_{4}\right)$ is unstable for all $\tau>0$.

Notice that these roots changes as $\tau$ varies. Now, we determine whether there exists a critical delay $\tau_{c}>0$ interms of $d_{1}, d_{2}, d_{3}, k_{1}, k_{2}, k_{3}$ so that $\operatorname{Re}(\lambda)>0$ for $\tau>\tau_{c}$. This critical value of $\tau$ is the point at which (3.10) transform from having a non positive to a non negative real parts. In other words, $\tau_{c}$ is the value of $\tau$ such that $\operatorname{Re}(\lambda)=0$, at which the transition from stability to instability occurs. By determining the conditions on the 
parameters to ensure that the steady state $\left(\psi / a_{1}, 0, \gamma / b_{4}\right)$ of the delay system (3.2) is still stable, we consider equation (3.10) as a complex variable mapping problem.

For this to occur considering the steady state $\left(\psi / a_{1}, 0, \gamma / b_{4}\right)$, there must be a boundary case, a critical value of $\tau$ such that the characteristic has a purely imaginary root. To determine whether or not such a $\tau$ exists, we reduce (3.10) to a polynomial problem and then seek for particular types of roots, thereby determining if a bifurcation can occur at such delay. We begin by looking for purely imaginary roots, and letting $\lambda(\tau)=\phi(\tau)+$ $i \sigma(\tau)$, where $\phi$ amd $\sigma$ are real, we have $\phi(0)<0$. By continuity of $\phi, \phi(\tau)<0$ for values of $\tau$ such that $0 \leq$ $\tau \leq \tau_{c}$ for some $\tau_{c}>0$. Therefore, the steady state remains stable for values of $\tau$. Substituting $\lambda(\tau)$ into equation (3.10) i.e.,

$$
\lambda^{3}+d_{1} \lambda^{2}+d_{2} \lambda+d_{3}+\left(k_{1} \lambda^{2}+k_{2} \lambda+k_{3}\right) e^{-\tau \lambda}=0
$$

we obtain

$$
\begin{gathered}
(\phi+i \sigma)^{3}+d_{1}(\phi+i \sigma)^{2}+d_{2}(\phi+i \sigma)+d_{3} \\
+\left(k_{1}(\phi+i \sigma)^{2}+k_{2}(\phi+i \sigma)+k_{3}\right) e^{-\tau(\phi+i \sigma)}=0 \\
\phi^{3}+3 \phi^{2} i \sigma-3 \phi \sigma^{2}-i \sigma^{3}+d_{1}\left(\phi^{2}+2 \phi i \sigma-\sigma^{2}\right)+d_{2}(\phi+i \sigma)+d_{3} \\
+\left[k_{1}\left(\phi^{2}+2 \phi i \sigma-\sigma^{2}\right)+k_{2}(\phi+i \sigma)+k_{3}\right] e^{-\tau(\phi+i \sigma)}=0 \\
\phi^{3}+3 \phi^{2} i \sigma-3 \phi \sigma^{2}-i \sigma^{3}+d_{1} \phi^{2}+2 d_{1} \phi i \sigma-d_{1} \sigma^{2}+d_{2} \phi+d_{2} i \sigma+d_{3} \\
+\left[k_{1} \phi^{2}+2 k_{1} \phi i \sigma-k_{1} \sigma^{2}+k_{2} \phi+k_{2} i \sigma+k_{3}\right] e^{-\tau(\phi+i \sigma)}=0 .
\end{gathered}
$$

We probably write the exponential in terms of trigonometric function, that is

But

$$
\begin{gathered}
e^{-\tau(\phi+i \sigma)}=e^{-\tau \phi} \cdot e^{-\tau i \sigma}, \text { ent } \\
e^{-\tau i}=\cos \tau \sigma-i \sin \tau \sigma, \\
\Rightarrow e^{-\tau \phi} \cdot e^{-\tau i \sigma}=e^{-\tau \phi}(\cos \tau \sigma-i \sin \tau \sigma) .
\end{gathered}
$$

Then we break the polynomial up into its real and imaginary parts, and write the exponential in terms of the trigonometric functions to get

$$
\begin{gathered}
d_{3}+d_{2} \phi+d_{1} \phi^{2}+\phi^{3}-d_{1} \sigma^{2}-3 \phi \sigma^{2}+i\left(d_{2} \sigma+2 d_{1} \phi \sigma+3 \phi^{2} \sigma-\sigma^{3}\right) \\
+e^{-\tau \phi}(\cos \tau \sigma-i \sin \tau \sigma)\left[k_{1} \phi^{2}+2 k_{1} \phi i \sigma-k_{1} \sigma^{2}+k_{2} \phi+k_{2} i \sigma+k_{3}\right]=0 \\
d_{3}+d_{2} \phi+d_{1} \phi^{2}+\phi^{3}-d_{1} \sigma^{2}-3 \phi \sigma^{2}+i\left(d_{2} \sigma+2 d_{1} \sigma+3 \phi^{2} \sigma-\sigma^{3}\right) \\
+e^{-\tau \phi}\left[\cos \tau \sigma\left(k_{1} \phi^{2}+2 k_{1} \phi i \sigma-k_{1} \sigma^{2}+k_{2} \phi+k_{2} i \sigma+k_{3}\right)\right] \\
-e^{-\tau \phi}\left[i \sin \tau \sigma\left(k_{1} \phi^{2}+2 k_{1} \phi i \sigma-k_{1} \sigma^{2}+k_{2} \phi+k_{2} i \sigma+k_{3}\right)\right]=0 \\
d_{3}+d_{2} \phi+d_{1} \phi^{2}+\phi^{3}-d_{1} \sigma^{2}-3 \phi \sigma^{2}+i\left(d_{2} \sigma+2 d_{1} \phi \sigma+3 \phi^{2} \sigma-\sigma^{3}\right) \\
+e^{-\tau \phi}\left[\left(k_{3}+k_{2} \phi+k_{1} \phi^{2}-k_{1} \sigma^{2}\right) \cos \tau \sigma+i\left(k_{2} \sigma+2 k_{1} \sigma\right) \cos \tau \sigma\right] \\
+e^{-\tau \phi}\left[\left(-k_{1} \sigma^{2}+k_{3}+k_{2} \phi+\phi\right)(-i \sin \tau)+i\left(k_{2} \sigma+2 k_{1} \phi \sigma\right)(-i \sin \tau)\right]=0 \\
d_{3}+d_{2} \phi+d_{1} \phi^{2}+\phi^{3}-d_{1} \sigma^{2}-3 \phi \sigma^{2}+i\left(d_{2} \sigma+2 d_{1} \phi \sigma+3 \phi^{2} \sigma-\sigma^{3}\right) \\
+e^{-\tau \phi}\left[\left(k_{2} \sigma+2 k_{1} \phi \sigma\right) \sin \tau \sigma+\left(k_{3}+k_{2} \phi+k_{1} \phi^{2}-k_{1} \sigma^{2}\right) \cos \tau \sigma\right] \\
+i e^{-\tau \phi}\left[\left(k_{1} \sigma^{2}-k_{3}-k_{2} \phi-k_{1} \phi^{2}\right) \sin \tau \sigma+\left(k_{2} \sigma+2 k_{1} \phi \sigma\right) \cos \tau \sigma\right]=0 .
\end{gathered}
$$


Assuming, we let $\phi\left(\tau_{c}\right)=0$ for some $\tau_{c}>0$ and $\phi(\tau)<0$ for $0 \leq \tau \leq \tau_{c}$, then the steady state $\left(\psi / a_{1}, 0, \gamma / b_{4}\right)$ may loss stability at $\tau=\tau_{c}$ or $\lambda\left(\tau_{c}\right)=i \sigma\left(\tau_{c}\right)$. In fact $i \sigma$ is a root of equation (4.26) if and only if we take terms of $\sigma$ only and the constants.

$$
-i \sigma^{3}-d_{1} \sigma^{2}+d_{2} i \sigma+d_{3}+\left(-k_{1} \sigma^{2}+k_{2} i \sigma+k_{3}\right)(\cos \tau \sigma-i \sin \tau \sigma)=0
$$

In order for (3.12) to hold, both the real and imaginary parts must be zero. By equating real parts and imaginary parts of the right side of system (3.12) to zero, we have the equations;

$$
\begin{gathered}
k_{2} \sigma \sin \tau \sigma+\left(k_{3}-k_{1} \sigma^{2}\right) \cos \tau \sigma-d_{1} \sigma^{2}+d_{3}=0 \\
k_{2} i \sigma \cos \tau \sigma-\left(k_{3}-k_{1} \sigma^{2}\right) i \sin \tau \sigma-i \sigma^{3}+d_{2} i \sigma=0
\end{gathered}
$$

which gives;

$$
\begin{aligned}
& k_{2} \sigma \sin \tau \sigma+\left(k_{3}-k_{1} \sigma^{2}\right) \cos \tau \sigma=d_{1} \sigma^{2}-d_{3} \\
& k_{2} i \sigma \cos \tau \sigma-\left(k_{3}-k_{1} \sigma^{2}\right) i \sin \tau \sigma=i \sigma^{3}-d_{2} i \sigma
\end{aligned}
$$

Squaring both sides of (4.29) and (4.30) one obtains;

$$
\begin{gathered}
\left(k_{2} \sigma \sin \tau \sigma\right)^{2}+\left(k_{3}-k_{1} \sigma^{2}\right)^{2}(\cos \tau \sigma)^{2}=\left(d_{1} \sigma^{2}-d_{3}\right)^{2} \\
\Rightarrow k_{2}^{2} \sigma^{2} \sin ^{2} \tau \sigma+\left(k_{3}^{2}-2 k_{1} k_{3} \sigma^{2}+k_{1}^{2} \sigma^{4}\right) \cos ^{2} \tau \sigma=d_{1}^{2} \sigma^{4}-2 d_{1} d_{3} \sigma^{2}+d_{3}^{2} \\
\left(k_{2} \sigma \cos \tau \sigma\right)^{2}+\left(k_{3}-k_{1} \sigma^{2}\right)^{2}(-\sin \tau \sigma)^{2}=\left(\sigma^{3}-d_{2} \sigma\right)^{2} \\
\Rightarrow k_{2}^{2} \sigma^{2} \cos ^{2} \tau \sigma+\left(k_{3}^{2}-2 k_{1} k_{3} \sigma^{2}+k_{1}^{2} \sigma^{4}\right) \sin ^{2} \tau \sigma=\sigma^{6}-2 d_{2} \sigma^{4}+d_{2}^{2} \sigma^{2}
\end{gathered}
$$

Adding up the squares of (3.15) and (3.16) we have

$$
\begin{gathered}
k_{2}^{2} \sigma^{2}\left(\sin ^{2} \tau \sigma+\cos ^{2} \tau \sigma\right)+\left[\left(k_{3}^{2}-2 k_{1} k_{3} \sigma^{2}+k_{1}^{2} \sigma^{4}\right)\left(\sin ^{2} \tau \sigma+\cos ^{2} \tau \sigma\right)\right] \\
=d_{1}^{2} \sigma^{4}-2 d_{1} d_{3} \sigma^{2}+d_{3}^{2}+\sigma^{6}-2 d_{2} \sigma^{4}+d_{2}^{2} \sigma^{2}
\end{gathered}
$$

But $\sin ^{2} \tau \sigma+\cos ^{2} \tau \sigma=1$, then we have;

$$
\begin{array}{r}
k_{2}^{2} \sigma^{2}+k_{3}^{2}-2 k_{1} k_{3} \sigma^{2}+k_{1}^{2} \sigma^{4}=d_{1}^{2} \sigma^{4}-2 d_{1} d_{3} \sigma^{2}+d_{3}^{2}+\sigma^{6}-2 d_{2} \sigma^{4}+d_{2}^{2} \sigma^{2} \\
\Rightarrow U(\sigma)=\sigma^{6}+\left(d_{1}^{2}-2 d_{2}-k_{1}^{2}\right) \sigma^{4}+\left(d_{2}^{2}-2 d_{1} d_{3}+2 k_{1} k_{3}-k_{2}^{2}\right) \sigma^{2}+d_{3}^{2}-k_{3}^{2}=0
\end{array}
$$

After squaring (3.13) and (3.14) and then summed (3.15) and (3.16), we observe two things. The first observation is that the trigonometric term disappears from the polynomial and the delay, $\tau$, has been eliminated. The second observation tells us that the equation is an even polynomial. This is because when an even or odd function is squared, it will always result to an even polynomial, that is $s(-\sigma)^{2}=( \pm s(\sigma))^{2}=s(\sigma)^{2}$ For further simplification of (4.33), we define a new variables by letting ;

$$
\left(g \equiv \sigma^{2}, \quad h \equiv d_{1}^{2}-2 d_{2}-k_{1}^{2}, \quad l \equiv d_{2}^{2}-2 d_{1} d_{3}+2 k_{1} k_{3}-k_{2}^{2}, \quad m_{1} \equiv d_{3}^{2}-k_{3}^{2}\right) \in \mathbb{R}
$$

Then we have equation (4.33) written as

$$
N(g)=g^{3}+h g^{2}+l g+m_{1}=0
$$

where $N$ is a polynomial. Remember that we are interested in $\sigma \in \mathbb{R}$, therefore, if all the real part of $N$ are non positive, it simply implies that there can be no simultaneous solution of (3.13) and (3.14). Alternatively, if there is a non negative real root $g$ to $N$, there is a delay $\tau$ corresponding to $\sigma$ which solves equations (3.13) and (314).

\section{Lemma 3:}

Assume that equation (3.18) has negative real roots, then all roots of equation (3.10) have negative real parts if $\tau>0$. 


\section{Proof:}

As far as equation (3.18) has negative real roots, any real number $\sigma$ is not a root of equation (3.17). This ensures that any real number $\sigma$ is not a root of equation (3.12). Hence for any real number $\sigma$, the value $i \sigma$ is not a root of equation (3.10) and this implies that there is no $\tau_{c}$ such that $\lambda\left(\tau_{c}\right)=i \sigma\left(\tau_{c}\right)$ is a root of equation (3.11). Because of proposition 2, for a root of (3.10) corresponding to $\tau=0, \operatorname{Re}(\lambda(0))<0$. Since $\operatorname{Re}(\lambda(\tau))$ is a continuous function of $\tau$, we conclude that all roots of (3.10) have negative real parts. Since (3.18) is odd (of degree three), we are guaranteed a negative real root. The only way to have a simple positive real root in this case is to have two positive real roots.

To present the conditions that ensure equation (3.18) has a positive real root or has no positive real roots, we take the Sturm chain of the polynomial (3.18) denoted by

$$
\begin{aligned}
& N_{0}(g)=N(g) ; \\
& N_{1}(g)=N^{\prime}(g) .
\end{aligned}
$$

Therefore, $N_{0}(g)=N(g)=g^{3}+h g^{2}+l g+m_{1}=0$

$$
N_{1}(g)=N^{\prime}(g)=3 g^{2}+2 h g+l=0
$$

The roots of (3.19) are

$$
g_{1}=\frac{-h+\sqrt{h^{2}-3 l}}{3}, g_{2}=\frac{-h-\sqrt{h^{2}-3 l}}{3} .
$$

One of these is positive if $h<0$ or $h>0$ and $l<0$, so either $h$ or $l$ must be negative. So we have;

\section{Lemma 4:}

A steady state with characteristic equation (3.18) is stable in the absence of delay and becomes unstable with increasing delay if and only if $h, l$ and $m$ are not all positive and

1. $d_{1}+k_{1}>0, d_{3}+k_{3}>0,\left(d_{1}+k_{1}\right)\left(d_{2}+k_{2}\right)-\left(d_{3}+k_{3}\right)>0$

2. Suppose that either (a) $m<0$, or (b) $m \geq 0, h^{2}-3 l>0, h<0$ and $N\left(g_{1}\right)<0$ then equation (3.18) has positive real roots.

3. Suppose $m \geq 0$ and $h^{2}-3 l>0$, then equation (3.18) has no positive real roots.

4.

\section{Proof:}

1. $d_{1}=2 a_{1}+b_{4}+\left(b_{1}+b_{2}\right) \frac{\gamma}{b_{4}}$

$k_{1}=-\zeta-\frac{\beta a_{3} \psi}{a_{1} \mu}$

$d_{2}=\left(a_{1}+\left(b_{1}+b_{2}\right) \frac{\gamma}{b_{4}}\right)\left(a_{1}+b_{4}\right)+a_{1} b_{4}$

$k_{2}=-\left(\zeta+\frac{\beta a_{3} \psi}{a_{1} \mu}\right)\left(a_{1}+b_{4}\right)$,

$d_{3}=a_{1} b_{4}\left(a_{1}+\left(b_{1}+b_{2}\right) \frac{\gamma}{b_{4}}\right)$,

$k_{3}=-a_{1} b_{4}\left(\zeta+\frac{\beta a_{3} \psi}{a_{1} \mu}\right)$.

Suppose that the Routh-Hurwitz criterion holds. That is if $d_{1}+k_{1}>0, d_{3}+k_{3}>0$ and $\left(d_{1}+k_{1}\right)\left(d_{2}+\right.$ $\left.k_{2}\right)-\left(d_{3}+k_{3}\right)>0$, then the characteristic equation (3.18) is stable in the absence of delay.

(i) If (a) is true, that is if $m<0$, then from equation (3.18) we have that $N(0)=m<0$. Also, we observe that

$$
\lim _{g \rightarrow \infty} N(g)=\infty
$$

and this implies that the intermediate value theorem, guarantees that equation (3.18) must have a positive real root $t_{0}$, with $N\left(t_{0}\right)=0$. 
2. Now, suppose that (b) is true, that is if $m \geq 0$ and $h^{2}-3 l<0$ and $h>0$, we find that $g_{1}$ is real and $g_{1}>0$. Since $N(0)=m \geq 0$ and $N\left(g_{1}\right)<0$, also by the intermediate value theorem, $N$ has a zero between the origin and $g_{1}$.

Notice that $N^{\prime}(g)=0$ has no real root since both zeros $g_{1}$ and $g_{2}$ of $N^{\prime}$ are not real if $3 l>h^{2}$.

Therefore,

$$
N^{\prime}(0)=l>\frac{h^{2}}{3} \geq 0
$$

In conclusion, we have that the coefficients; $h, l$ and $m$ of the quadratic polynomial $N^{\prime}$ are all positive real numbers and it simply means that $N$ is increasing on the real numbers. Actually, because $N(0)=m \geq 0$, we observe that when $m>0, N(g)$ does not vanish and thus, equation (3.18) does not have any positive real root. Also, lemma 4(ii) simply tells us that there is $\sigma$ such that $\sigma$ is a solution of the characteristic equation (3.10), thus indicating that real parts of all the eigenvalues of $\mathrm{H}$ are non positive for all delay $\tau \geq 0$. The summary of the above analysis is represented in the theorems below.

\section{Theorem 5:}

The uninfected steady state $\left(\psi / a_{1}, 0, \gamma / b_{4}\right)$ of the delay system (3.2) is asymptotically stable for all $\tau \geq 0$ if $\zeta+\frac{\beta a_{3} \psi}{a_{1} \mu}<a_{1}+\frac{\left(b_{1}+b_{2}\right) \gamma}{b_{4}}, a_{1}, a_{3}, b_{1}, b_{2}, b_{4}, \gamma, \zeta, \beta, \psi, \mu>0, m \geq 0$ and $h^{2}-3 l<0$.

\section{Proof:}

By part (ii) of lemma 4, equation (3.18) has no positive roots. Lemma 3 ensures that all roots of (3.10) have negative real parts for $\tau>0$. Therefore, the steady state $\left(\psi / a_{1}, 0, \gamma / b_{4}\right)$ of the delay system (3.2) is asymptotically stable for all $\tau \geq 0$. We will introduce the Hopf Bifurcation theorem (Hassard et al, 1981), as shall be discussed below.

To establish Hopf bifurcation at $\tau=\tau_{c}$, we need to show that

$$
\frac{d}{d \tau} \phi(\tau) \neq 0
$$

Now to ensure that Hopf bifurcation occurs, we provide conditions on the parameters. Suppose the conditions in Lemma 4(ai) hold, then equation (3.17) has a positive root. We denote by $g_{j}, j \in\{1\}$ or $j \in\{1,2,3\}$ depending on the number of positive roots that equation (3.18) has. Observe that equation (3.17) has up to six roots i.e., $\pm \sqrt{g_{j}}$ for $j=1,2,3$.

Notice that if the solution of equation (3.9) exists, it is among these $\pm \sqrt{g_{j}}$ for $j=1,2,3$ and that if $\lambda=i \sigma$ is a root of equation (3.10), $-i \sigma$ will also be a root.

To equations (3.13) and (3.14) we substitute $\sigma_{j}$ and then solve for $\tau$, which will then be of the form

where $j=0,1,2$.

$$
\tau_{j}^{(n)}=\frac{1}{\sigma_{j}} \sin ^{-1}\left[\frac{k_{1} \sigma_{j}^{5}+\left(d_{1} k_{2}-k_{3}-d_{2} k_{1}\right) \sigma_{j}^{3}+\left(d_{1} k_{3}-d_{3} k_{2}\right) \sigma_{j}}{k_{2}^{2} \sigma_{j}^{2}+\left(k_{3}-k_{1} \sigma^{2}\right)^{2}}\right]+\left[\frac{2 \pi(n-1)}{\sigma_{j}}\right]
$$

if we multiply (3.13) by $k_{2} \sigma_{c}$ and (3.14) by $k_{3}-k_{1} \sigma_{c}^{2}$ and also add the resulting product, we obtain $k_{2}^{2} \sigma_{c}^{2} \sin \sigma_{c} \tau_{c}+k_{2} \sigma_{c}\left(k_{3}-k_{1} \sigma_{c}^{2}\right) \cos \sigma_{c} \tau_{c}=k_{2} \sigma_{c}\left(d_{1} \sigma_{c}^{2}-d_{3}\right)$

$$
\begin{aligned}
& -k_{2} \sigma_{c}\left(k_{3}-k_{1} \sigma_{c}^{2}\right) \cos \sigma_{c} \tau_{c}+\left(k_{3}-k_{1} \sigma_{c}^{2}\right)^{2} \sin \sigma_{c} \tau_{c}=-\left(k_{3}-k_{1} \sigma_{c}^{2}\right)\left(\sigma_{c}^{3}-d_{2} \sigma_{c}\right) \\
& \Rightarrow\left[k_{2}^{2} \sigma_{c}^{2}+\left(k_{3}-k_{1} \sigma_{c}^{2}\right)^{2}\right] \sin \sigma_{c} \tau_{c}=k_{2} \sigma_{c}\left(d_{1} \sigma_{c}^{2}-d_{3}\right)-\left(k_{3}-k_{1} \sigma_{c}^{2}\right)\left(\sigma_{c}^{3}-d_{2} \sigma_{c}\right)
\end{aligned}
$$


International Journal of Trend in Scientific Research and Development (IJTSRD) ISSN: 2456-6470

$$
\begin{gathered}
\sin \sigma_{c} \tau_{c}=\frac{k_{2} \sigma_{c}\left(d_{1} \sigma_{c}^{2}-d_{3}\right)-\left(k_{3}-k_{1} \sigma_{c}^{2}\right)\left(\sigma_{c}^{3}-d_{2} \sigma_{c}\right)}{\left[k_{2}^{2} \sigma_{c}^{2}+\left(k_{3}-k_{1} \sigma_{c}^{2}\right)^{2}\right]} \\
\tau_{c}=\frac{1}{\sigma_{j}} \sin ^{-1}\left[\frac{k_{1} \sigma_{j}^{5}+\left(d_{1} k_{2}-k_{3}-d_{2} k_{1}\right) \sigma_{j}^{2}+\left(d_{2} k_{3}-d_{3} k_{2}\right) \sigma_{j}}{k_{2}^{2} \sigma_{j}^{2}+\left(k_{3}-k_{1} \sigma^{2}\right)^{2}}\right]+\left[\frac{2 \pi(n-1)}{\sigma_{j}}\right] \\
\tau_{j}^{(n)}=\frac{1}{\sigma_{j}} \sin ^{-1}\left[\frac{k_{1} \sigma_{j}^{5}+\left(d_{1} k_{2}-k_{3}-d_{2} k_{1}\right) \sigma_{j}^{2}+\left(d_{2} k_{3}-d_{3} k_{2}\right) \sigma_{j}}{k_{2}^{2} \sigma_{j}^{2}+\left(k_{3}-k_{1} \sigma^{2}\right)^{2}}\right]+\left[\frac{2 \pi(n-1)}{\sigma_{j}}\right] .
\end{gathered}
$$

Now let $\tau_{c}$ be the smallest of such $\tau$ for which $\phi\left(\tau_{c}\right)=0$.

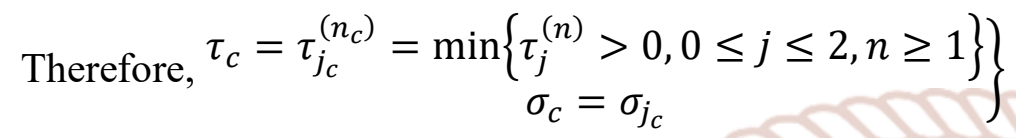

\section{Theorem 6:}

Let the critical time lag $\tau_{c}$ and $\sigma_{c}$ be defined as (3.21) for the time lag $\tau$ and suppose that $3 \sigma_{c}^{6}+2 h \sigma_{c}^{4}+l \sigma_{c}^{2} \neq$ 0 then the system of delay differential equation (3.2) exhibits the Hopf bifurcation at the steady state $\left(\psi / a_{1}, 0, \gamma / b_{4}\right)$.

\section{Proof:}

To establish Hopf bifurcation, we show that

$$
\left.\frac{d \phi(\tau)}{d \tau}\right|_{\tau=\tau_{c}} \neq 0
$$

and this guarantees that Hopf bifurcation occurs. For (3.21) to hold, both the real and imaginary parts of (3.11) must be zero. So we get the pair of equations

$$
\begin{aligned}
& \quad e^{-\tau}\left[\left(k_{2} \sigma+2 k_{1} \phi \sigma\right) \sin \phi \sigma+\left(k_{3}+k_{2} \phi-k_{1} \phi^{2}-k_{1} \sigma^{2}\right) \cos \phi \sigma\right] \\
& +d_{3}+d_{2} \phi+d_{1} \phi^{2}+\phi^{3}-d_{1} \sigma^{2}-3 \phi \sigma^{2}=0 \\
& \quad i e^{-\tau}\left[\left(k_{1} \sigma^{2}-k_{3}-k_{2} \phi-k_{1} \phi^{2}\right) \sin \phi \sigma+\left(k_{2} \sigma+2 k_{1} \phi \sigma\right) \cos \phi \sigma\right] \\
& +i\left(d_{2} \sigma+2 d_{1} \phi \sigma+3 \phi^{2} \sigma-\sigma^{3}\right)=0
\end{aligned}
$$

which finally gives

$$
\begin{aligned}
& e^{-\tau \phi}\left[\left(k_{2} \sigma+2 k_{1} \phi \sigma\right) \sin \phi \sigma+\left(k_{3}+k_{2} \phi-k_{1} \phi^{2}-k_{1} \sigma^{2}\right) \cos \phi \sigma\right] \\
& =\left(d_{1}+3 \phi\right) \sigma^{2}-d_{3}-d_{2} \phi-d_{1} \phi^{2}-\phi^{3} \\
& e^{-\tau \phi}\left[\left(k_{1} \sigma^{2}-k_{3}-k_{2} \phi-k_{1} \phi^{2}\right) \sin \phi \sigma+\left(k_{2} \sigma+2 k_{1} \phi \sigma\right) \cos \phi \sigma\right] \\
& =\sigma^{3}-d_{2} \sigma-2 d_{1} \phi \sigma-3 \phi^{2} \sigma
\end{aligned}
$$

From equation (3.25), we have that $e^{-\tau \phi}=e^{0}$ since $\tau \phi=0$. Then $e^{0}=1$, and selecting $\sigma$ from the equation we obtain

$$
k_{2} \sigma \sin \phi \sigma+k_{3} \cos \phi \sigma-k_{1} \sigma^{2} \cos \phi \sigma=d_{1} \sigma^{2}-d_{3}
$$

We at this moment differentiate equation (3.27) with respect to $\tau$ after which we evaluate it at $\tau=\tau_{c}$ for which $\phi\left(\tau_{c}\right)=0$ and $\sigma\left(\tau_{c}\right)=\sigma_{c}$ and get

$$
\begin{gathered}
\left.\frac{d \sigma}{d \tau}\right|_{\tau=\tau_{c}}=k_{2} \sin \sigma_{c} \tau_{c}+\tau_{c} k_{2} \sigma_{c} \cos \sigma_{c} \tau_{c}-k_{3} \tau_{c} \sin \sigma_{c} \tau_{c}-2 k_{1} \sigma_{c} \cos \sigma_{c} \tau_{c}+\tau_{c} k_{1} \sigma_{c}^{2} \sin \sigma_{c} \tau_{c} \\
=2 d_{1} \sigma_{c} \\
\Longrightarrow 2 d_{1} \sigma_{c}+\left(2 k_{1} \sigma_{c}-\tau_{c} k_{2} \sigma_{c}\right) \cos \sigma_{c} \tau_{c}+\left(k_{3} \tau_{c}-\tau_{c} k_{1} \sigma_{c}{ }^{2}-k_{2}\right) \sin \sigma_{c} \tau_{c}=A_{1}
\end{gathered}
$$


International Journal of Trend in Scientific Research and Development (IJTSRD) ISSN: 2456-6470

Select $\phi$ from (3.25), differentiate with respect to $\tau$ and evaluating at $\tau=\tau_{c}$ for which $\phi\left(\tau_{c}\right)=0$ and $\sigma\left(\tau_{c}\right)=$ $\sigma_{c}$, we then obtain

$$
2 k_{1} \phi \sigma \sin \phi \sigma+k_{2} \phi \cos \phi \sigma+k_{1} \phi^{2} \cos \phi \sigma=3 \phi \sigma^{2}-d_{2} \phi-d_{1} \phi^{2}-\phi^{3}
$$

$$
\begin{gathered}
\left.\frac{d \phi}{d \tau}\right|_{\tau=\tau_{c}}=2 k_{1} \sigma \sin \sigma_{c} \tau_{c}+2 \tau_{c} \phi k_{1} \sigma_{c} \cos \sigma_{c} \tau_{c}+k_{2} \cos \sigma_{c} \tau_{c}-\tau_{c} k_{2} \phi \sin \sigma_{c} \tau_{c}+2 k_{1} \phi \cos \sigma_{c} \tau_{c} \\
-k_{1} \phi^{2} \tau_{c} \sin \sigma_{c} \tau_{c}=3{\sigma_{c}}^{2}-d_{2}-2 d_{1} \phi-3 \phi^{2}
\end{gathered}
$$

But $\phi\left(\tau_{c}\right)=0$

$$
\Rightarrow 3 \sigma_{c}^{2}-d_{2}-2 k_{1} \sigma \sin \sigma_{c} \tau_{c}-k_{2} \cos \sigma_{c} \tau_{c}=A_{1}
$$

Also from equation (3.26) we have $e^{\tau \phi}=e^{0}=1$. Selecting $\sigma$ from (3.26) and differentiating with respect to $\tau$ and evaluating at $\tau=\tau_{c}$ for which $\phi\left(\tau_{c}\right)=0$ and $\sigma\left(\tau_{c}\right)=\sigma_{c}$ we get

$$
k_{1} \sigma^{2} \sin \sigma \tau-k_{3} \sin \sigma \tau+k_{2} \sigma \cos \sigma \tau=\sigma^{3}-d_{2} \sigma
$$

Then,

$$
\begin{gathered}
\left.\frac{d \sigma}{d \tau}\right|_{\tau=\tau_{c}}=2 k_{1} \sigma_{c} \sin \sigma_{c} \tau_{c}+k_{1} \tau_{c} \sigma_{c}^{2} \cos \sigma_{c} \tau_{c}-\tau_{c} k_{3} \cos \sigma_{c} \tau_{c}-k_{2} \tau_{c} \sigma_{c} \sin \sigma_{c} \tau_{c}+k_{2} \cos \sigma_{c} \tau_{c} \\
=3 \sigma_{c}^{2}-d_{2} \\
\Rightarrow d_{2}-3 \sigma_{c}{ }^{2}+\left(k_{2}+k_{1} \tau_{c} \sigma_{c}^{2}-\tau_{c} k_{3}\right) \cos \sigma_{c} \tau_{c}+\left(2 k_{1} \sigma_{c}-k_{2} \tau_{c} \sigma_{c}\right) \sin \sigma_{c} \tau_{c}=A_{2}
\end{gathered}
$$

Again, selecting $\phi$ from (3.26), then and differentiating with respect to $\tau$ and evaluating at $\tau=\tau_{c}$ for which $\phi\left(\tau_{c}\right)=0$ and $\sigma\left(\tau_{c}\right)=\sigma_{c}$ we have

$$
-k_{2} \phi \cos \sigma \tau-k_{1} \phi^{2} \sin \sigma \tau+2 k_{1} \phi \sigma \cos \sigma \tau=-2 d_{1} \phi \sigma-3 \phi^{3} \sigma
$$

Then,

$$
\begin{gathered}
\left.\frac{d \phi}{d \tau}\right|_{\tau=\tau_{c}}=-k_{2} \sin \sigma_{c} \tau_{c}-k_{2} \tau_{c} \phi_{c} \cos \sigma_{c} \tau_{c}-2 k_{1} \phi_{c} \sin \sigma_{c} \tau_{c}-k_{1} \tau_{c} \phi_{c}{ }^{2} \sin \sigma_{c} \tau_{c} \\
+2 k_{1} \sigma_{c} \cos \sigma_{c} \tau_{c}-2 k_{1} \phi_{c} \sigma_{c} \sin \sigma_{c} \tau_{c}=-2 d_{1} \sigma_{c}-6 \phi_{c} \sigma_{c} \\
\Rightarrow-k_{2} \sin \sigma_{c} \tau_{c}+2 k_{1} \sigma_{c} \cos \sigma_{c} \tau_{c}=-2 d_{1} \sigma_{c} \\
\Rightarrow 2 d_{1} \sigma_{c}-k_{2} \sin \sigma_{c} \tau_{c}+2 k_{1} \sigma_{c} \cos \sigma_{c} \tau_{c}=A_{2}
\end{gathered}
$$

At this point, we solve for the difference of $A_{1}$ and $A_{2}$ and the sum of $A_{2}$ and $A_{1}$, that is $\left.A_{1} \frac{d \sigma}{d \tau}\right|_{\tau=\tau_{c}}-\left.A_{2} \frac{d \phi}{d \tau}\right|_{\tau=\tau_{c}}$ and $\left.A_{2} \frac{d \sigma}{d \tau}\right|_{\tau=\tau_{c}}+\left.A_{1} \frac{d \phi}{d \tau}\right|_{\tau=\tau_{c}}$ respectively, then we obtain

$$
\begin{gathered}
\left.A_{1} \frac{d \sigma}{d \tau}\right|_{\tau=\tau_{c}}-\left.A_{2} \frac{d \phi}{d \tau}\right|_{\tau=\tau_{c}} \\
\Rightarrow\left[2 d_{1} \sigma_{c}+\left(2 k_{1} \sigma_{c}-\tau_{c} k_{2} \sigma_{c}\right) \cos \sigma_{c} \tau_{c}+\left(k_{3} \tau_{c}-\tau_{c} k_{1} \sigma_{c}{ }^{2}-k_{2}\right) \sin \sigma_{c} \tau_{c}\right] \\
-\left[2 d_{1} \sigma_{c}-k_{2} \sin \sigma_{c} \tau_{c}+2 k_{1} \sigma_{c} \cos \sigma_{c} \tau_{c}\right] \\
\Rightarrow-\tau_{c} k_{2} \sigma_{c} \cos \sigma_{c} \tau_{c}+\left(k_{3} \tau_{c}-\tau_{c} k_{1} \sigma_{c}{ }^{2}\right) \sin \sigma_{c} \tau_{c}
\end{gathered}
$$

Since $\tau_{c}=\sigma_{c}$, we have

$$
\Rightarrow k_{2} \sigma_{c}^{2} \cos \sigma_{c} \tau_{c}+\left(k_{1} \sigma_{c}^{3}-k_{3} \sigma_{c}\right) \sin \sigma_{c} \tau_{c}
$$

Therefore,

Also

$$
A_{3}=k_{2} \sigma_{c}{ }^{2} \text { and } A_{4}=k_{1} \sigma_{c}{ }^{3}-k_{3} \sigma_{c}
$$

$$
\begin{gathered}
\left.A_{2} \frac{d \sigma}{d \tau}\right|_{\tau=\tau_{c}}+\left.A_{1} \frac{d \phi}{d \tau}\right|_{\tau=\tau_{c}} \\
\Rightarrow\left[d_{2}-3 \sigma_{c}{ }^{2}+\left(k_{2}+k_{1} \tau_{c} \sigma_{c}{ }^{2}-\tau_{c} k_{3}\right) \cos \sigma_{c} \tau_{c}+\left(2 k_{1} \sigma_{c}-k_{2} \tau_{c} \sigma_{c}\right) \sin \sigma_{c} \tau_{c}\right] \\
+\left[3 \sigma_{c}{ }^{2}-d_{2}-2 k_{1} \sigma \sin \sigma_{c} \tau_{c}-k_{2} \cos \sigma_{c} \tau_{c}\right]
\end{gathered}
$$


International Journal of Trend in Scientific Research and Development (IJTSRD) ISSN: 2456-6470

Again remember that $\tau_{c}=\sigma_{c}$, and we then obtain

$$
\begin{aligned}
& -k_{2} \sigma_{c}{ }^{2} \sin \sigma_{c} \tau_{c}+\left(k_{1} \sigma_{c}{ }^{3}-k_{3} \sigma_{c}\right) \cos \sigma_{c} \tau_{c} \\
& \Rightarrow k_{2} \sigma_{c}{ }^{2} \sin \sigma_{c} \tau_{c}-\left(k_{1} \sigma_{c}{ }^{3}-k_{3} \sigma_{c}\right) \cos \sigma_{c} \tau_{c}
\end{aligned}
$$

Therefore,

$$
A_{3}=k_{2} \sigma_{c}^{2} \text { and }-A_{4}=\left(k_{1} \sigma_{c}^{3}-k_{3} \sigma_{c}\right)
$$

The result of the differentiations, the difference and sum when put together, yields

$$
\begin{aligned}
& \left.A_{1} \frac{d \sigma}{d \tau}\right|_{\tau=\tau_{c}}-\left.A_{2} \frac{d \phi}{d \tau}\right|_{\tau=\tau_{c}}=A_{3} \cos \sigma_{c} \tau_{c}+A_{4} \sin \sigma_{c} \tau_{c} \\
& \left.A_{2} \frac{d \sigma}{d \tau}\right|_{\tau=\tau_{c}}+\left.A_{1} \frac{d \phi}{d \tau}\right|_{\tau=\tau_{c}}=A_{3} \sin \sigma_{c} \tau_{c}-A_{4} \cos \sigma_{c} \tau_{c}
\end{aligned}
$$

To solve equations (3.31) and (3.32) simultaneously, we multiply equation (3.31) by $A_{2}$ and equation (3.32) by $A_{1}$, then subtract the product of (3.31) from (3.32) and this eliminates $\frac{d \sigma}{d \tau}$. Therefore, we have

$$
\begin{gathered}
\left.A_{1} A_{2} \frac{d \sigma}{d \tau}\right|_{\tau=\tau_{c}}+\left.A_{1}^{2} \frac{d \phi}{d \tau}\right|_{\tau=\tau_{c}}=A_{1} A_{3} \sin \sigma_{c} \tau_{c}-A_{1} A_{4} \cos \sigma_{c} \tau_{c} \\
\left.A_{1} A_{2} \frac{d \sigma}{d \tau}\right|_{\tau=\tau_{c}}-\left.A_{2}^{2} \frac{d \phi}{d \tau}\right|_{\tau=\tau_{c}}=A_{2} A_{3} \cos \sigma_{c} \tau_{c}+A_{2} A_{4} \sin \sigma_{c} \tau_{c} \\
\left.\Rightarrow\left(A_{1}^{2}+A_{2}^{2}\right) \frac{d \phi}{d \tau}\right|_{\tau=\tau_{c}}=\left(A_{1} A_{3}-A_{2} A_{4}\right) \sin \sigma_{c} \tau_{c}-\left(A_{1} A_{4}+A_{2} A_{3}\right) \cos \sigma_{c} \tau_{c} \\
\left.\frac{d \phi}{d \tau}\right|_{\tau=\tau_{c}}=\frac{\left(A_{1} A_{3}-A_{2} A_{4}\right) \sin \sigma_{c} \tau_{c}-\left(A_{1} A_{4}+A_{2} A_{3}\right) \cos \sigma_{c} \tau_{c}}{A_{1}^{2}+A_{2}^{2}}
\end{gathered}
$$

Let us solve equations (3.13) and (3.14) simultaneously.

$$
\begin{aligned}
& k_{2} \sigma_{c} \sin \sigma_{c} \tau_{c}+\left(k_{3}-k_{1} \sigma_{c}^{2}\right) \cos \sigma_{c} \tau_{c}=d_{1} \sigma_{c}^{2}-d_{3} \\
& k_{2} \sigma_{c} \cos \sigma_{c} \tau_{c}-\left(k_{3}-k_{1} \sigma_{c}^{2}\right) \sin \sigma_{c} \tau_{c}=\sigma_{c}^{3}-d_{2} \sigma_{c}
\end{aligned}
$$

If we multiply (3.13) by $k_{3}-k_{1} \sigma_{c}^{2}$ and (3.14) by $k_{2} \sigma_{c}$ and add the resulting products, we obtain

$$
\begin{gathered}
k_{2} \sigma_{c}\left(k_{3}-k_{1} \sigma_{c}^{2}\right) \sin \sigma_{c} \tau_{c}+\left(k_{3}-k_{1} \sigma_{c}^{2}\right)^{2} \cos \sigma_{c} \tau_{c}=\left(d_{1} \sigma_{c}^{2}-d_{3}\right)\left(k_{3}-k_{1} \sigma_{c}^{2}\right) \\
k_{2}^{2} \sigma_{c}^{2} \cos \sigma_{c} \tau_{c}-k_{2} \sigma_{c}\left(k_{3}-k_{1} \sigma_{c}^{2}\right) \sin \sigma_{c} \tau_{c}=k_{2} \sigma_{c}\left(\sigma_{c}^{3}-d_{2} \sigma_{c}\right) \\
\Rightarrow k_{2}^{2} \sigma_{c}^{2} \cos \sigma_{c} \tau_{c}+\left(k_{3}-k_{1} \sigma_{c}^{2}\right)^{2} \cos \sigma_{c} \tau_{c}=\left(d_{1} \sigma_{c}^{2}-d_{3}\right)\left(k_{3}-k_{1} \sigma_{c}^{2}\right)+k_{2} \sigma_{c}\left(\sigma_{c}^{3}-d_{2} \sigma_{c}\right) \\
{\left[k_{2}^{2} \sigma_{c}^{2}+\left(k_{3}-k_{1} \sigma_{c}^{2}\right)^{2}\right] \cos \sigma_{c} \tau_{c}=\left(d_{1} \sigma_{c}^{2}-d_{3}\right)\left(k_{3}-k_{1} \sigma_{c}^{2}\right)+k_{2} \sigma_{c}\left(\sigma_{c}^{3}-d_{2} \sigma_{c}\right)} \\
\Rightarrow \cos \sigma_{c} \tau_{c}=\frac{k_{2} \sigma_{c}\left(\sigma_{c}^{3}-d_{2} \sigma_{c}\right)+\left(d_{1} \sigma_{c}^{2}-d_{3}\right)\left(k_{3}-k_{1} \sigma_{c}^{2}\right)}{\left[k_{2}^{2} \sigma_{c}^{2}+\left(k_{3}-k_{1} \sigma_{c}^{2}\right)^{2}\right]}
\end{gathered}
$$

On the other hand, if we multiply (3.13) by $k_{2} \sigma_{c}$ and (3.14) by $k_{3}-k_{1} \sigma_{c}^{2}$ and also add the resulting product, we obtain

$$
\begin{gathered}
k_{2}^{2} \sigma_{c}^{2} \sin \sigma_{c} \tau_{c}+k_{2} \sigma_{c}\left(k_{3}-k_{1} \sigma_{c}^{2}\right) \cos \sigma_{c} \tau_{c}=k_{2} \sigma_{c}\left(d_{1} \sigma_{c}^{2}-d_{3}\right) \\
-k_{2} \sigma_{c}\left(k_{3}-k_{1} \sigma_{c}^{2}\right) \cos \sigma_{c} \tau_{c}+\left(k_{3}-k_{1} \sigma_{c}^{2}\right)^{2} \sin \sigma_{c} \tau_{c}=-\left(k_{3}-k_{1} \sigma_{c}^{2}\right)\left(\sigma_{c}^{3}-d_{2} \sigma_{c}\right) \\
\Rightarrow\left[k_{2}^{2} \sigma_{c}^{2}+\left(k_{3}-k_{1} \sigma_{c}^{2}\right)^{2}\right] \sin \sigma_{c} \tau_{c}=k_{2} \sigma_{c}\left(d_{1} \sigma_{c}^{2}-d_{3}\right)-\left(k_{3}-k_{1} \sigma_{c}^{2}\right)\left(\sigma_{c}^{3}-d_{2} \sigma_{c}\right)
\end{gathered}
$$




$$
\sin \sigma_{c} \tau_{c}=\frac{k_{2} \sigma_{c}\left(d_{1} \sigma_{c}^{2}-d_{3}\right)-\left(k_{3}-k_{1} \sigma_{c}^{2}\right)\left(\sigma_{c}^{3}-d_{2} \sigma_{c}\right)}{\left[k_{2}^{2} \sigma_{c}^{2}+\left(k_{3}-k_{1} \sigma_{c}^{2}\right)^{2}\right]}
$$

Substituting (3.34) and (3.35) into equation (3.33) it yields (3.36) below

$$
\left.\frac{d \phi}{d \tau}\right|_{\tau=\tau_{c}}=\frac{3 \sigma_{c}^{6}+2 h \sigma_{c}^{4}+l \sigma_{c}^{2}}{A_{1}^{2}+A_{2}^{2}} \neq 0
$$

Therefore we conclude that Hopf bifurcation occurs when $\tau$ passes through the critical value which is assumed to be $\tau_{c}$.

\section{ANALYSIS OF RESULT}

Certainly, our interest is in determining if the steady state of the model system is stable or not. When the slightest disturbance completely alters the behavior of the system, them it is impossible to predict the long term evolution of the system under consideration. We therefore showed in this research work with the parameter value by Long et al (2008) that critical delay beyond which the steady state would lose its stability through oscillation does not exist.

Biologically, proposition (4i) states that if $a_{1}+$ $\frac{\left(b_{1}+b_{2}\right) \gamma}{b_{4}}>\zeta+\frac{\beta a_{3} \psi}{a_{1} \mu}$ then the steady state $\left(\frac{\psi}{a_{1}}, 0, \frac{\gamma}{b_{4}}\right)$ of system (4.18) is asymptotically stable and this implies that there will be viral clearance because, CTL will both cytolitically and non cytolitically take care of infected hepatocytes before they can produce sufficient amounts of viral progeny which finally results in the absence of the virus. This is shown in figure $(1 \mathrm{a}-1 \mathrm{c})$ and $(2 \mathrm{a}-2 \mathrm{c})$. Figure $1 \mathrm{~d}$ focused on the behavior of the density of hepatitis $B$ virus infected hepatocytes and CTL when the delay parameter $\tau=1$ and show the interaction between virus and immune response.
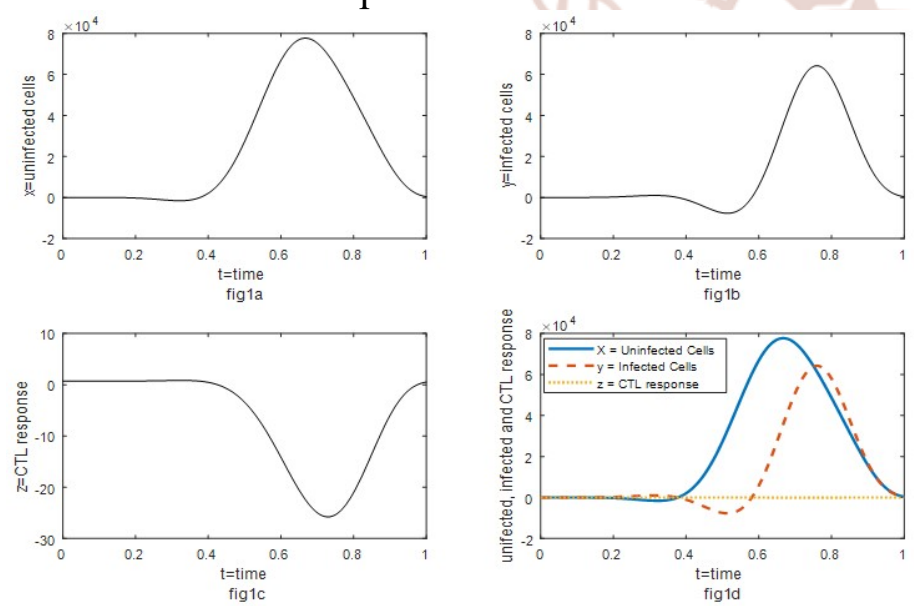

Figure $(1 \mathrm{a}-1 \mathrm{~d})$ : Numerical simulation of the delay model system (18) where parameter values, $a_{1}=$ $0.002, \gamma=1 E-3, a_{3}=1500, a_{4}=0.3, b_{1}=$ $500, b_{2}=1500, \psi=1, b_{4}=0.2, \zeta=0.04, \mu=$

$$
\begin{aligned}
0.58, \beta=10 & (\text { Long et al, 2008)and } \tau \\
& =1(\text { Eikenberry et al, 2009). }
\end{aligned}
$$
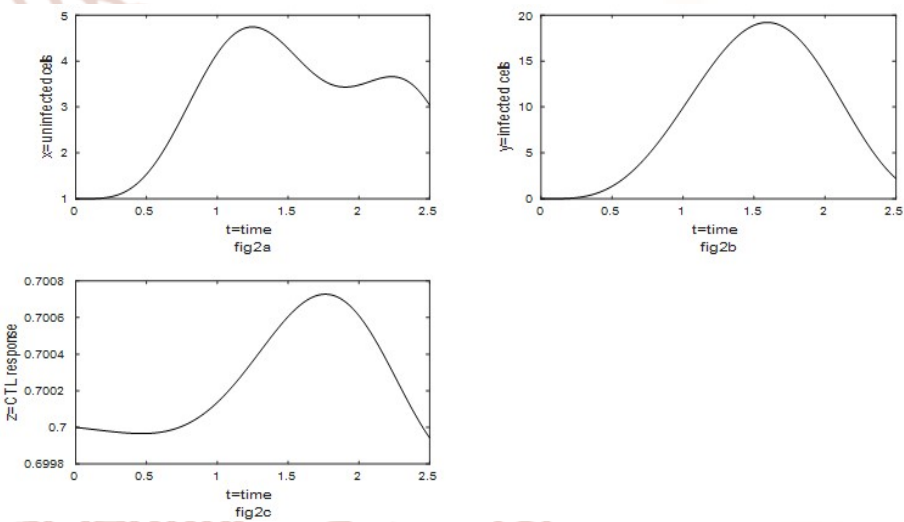

Figure $(2 \mathrm{a}-2 \mathrm{c})$ : Numerical simulation of the delay model system (18) where parameter values, $a_{1}=$ $0.002, \gamma=1 E-3, a_{3}=1500, a_{4}=0.3, b_{1}=$ $500, b_{2}=1500, \psi=1, b_{4}=0.2, \zeta=0.04, \mu=$ $0.58, \beta=10$ and $\tau=1$ with time ranging from 0 to

\section{5 .}
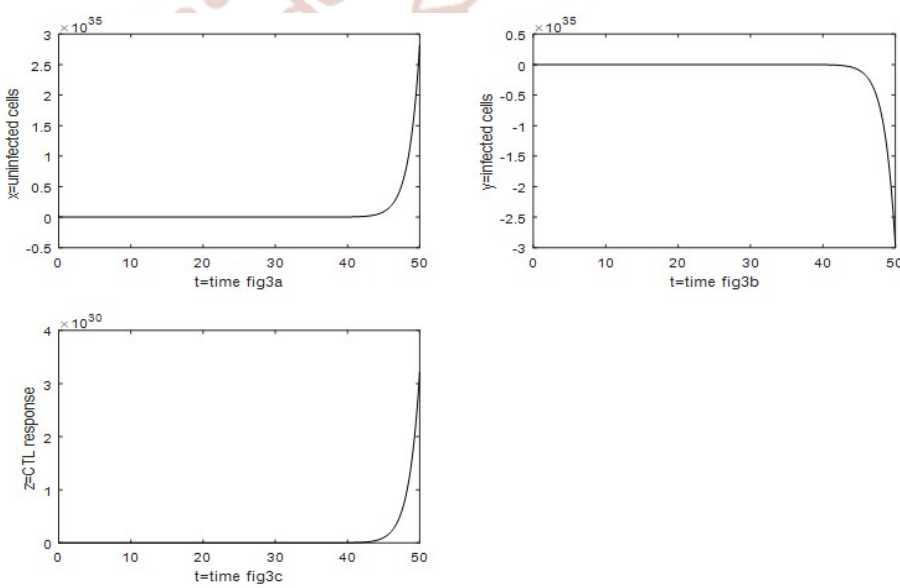

Figure $(3 a-3 c)$ : Numerical simulation of the delay model system (18) where parameter values, $a_{1}=$ $0.002, \gamma=1 E-3, a_{3}=1500, a_{4}=0.3, b_{1}=$ $500, b_{2}=1500, \psi=1, b_{4}=0.2, \zeta=0.04, \mu=$ $0.58, \beta=10$ and $\tau=1$ with time ranging from 0 to 50 
Figure $(1 \mathrm{a}-1 \mathrm{~d}),(2 \mathrm{a}-2 \mathrm{c})$ and $(3 \mathrm{a}-3 \mathrm{c})$ shows the delay effect on equation (3.2) with delay value $\tau=1$. The infected steady state of proposition (1i) of delay system (3.2) remains stable if the conditions in theorem 5 which states that the uninfected steady state $\left(\psi / a_{1}, 0, \gamma / b_{4}\right)$ of the delay system (3.2) is asymptotically stable for all $\tau \geq 0$ if $\zeta+\frac{\beta a_{3} \psi}{a_{1} \mu}<a_{1}+$ $\frac{\left(b_{1}+b_{2}\right) \gamma}{b_{4}}, a_{1}, a_{3}, b_{1}, b_{2}, b_{4}, \gamma, \zeta, \beta, \psi, \mu>0, m \geq$ 0 and $h^{2}-3 l<0$

are satisfied. Then, we can conclude that incorporating a discrete intracellular time delay, into system (3.1) does not change the stability of the steady state of proposition (1i). In this case, even if the immune response is maintained and virus persists, the virus may persist without causing much harm to the liver since the immune system is strongly responsive in eliminating the virus from within the hepatocytes without killing the hepatocytes.

If theorem 8 which says that suppose that $3 \sigma_{c}^{6}+$ $2 h \sigma_{c}^{4}+l \sigma_{c}^{2} \neq 0$ then the system of delay differential equation (4.18) exhibits the Hopf bifurcation at the steady state $\left(\frac{\psi}{a_{1}}, 0, \frac{\Upsilon}{b_{4}}\right)$ is satisfied, we have that the stability of the infected steady state of proposition (1i) of delay model equation (3.2) depends on the delay value and the delay can induce oscillation in the number of the uninfected hepatocytes, infected hepatocytes and CTL. If the individuals' ALT level is high, then, the virus may have escaped the control of the CTL and HBV infection is reactivated. However, if the immune control succeeds, the virus population will utterly be destroyed. Ciupe et al (2007), demonstrated that consideration of delayed cytotoxic and non cytotoxic immune reactions and the presence of cells refactory to infection was necessary to properly understand the dynamics of HBV acute infection and progression to chronic disease.

\section{SUMMARY AND CONCLUSION}

In summary, we have that clearance of acute HBV infection occurs subsequent to the appearance of the Cytotoxic T Lymphocyte (CTL) cells. The clearance may be cytolytic or noncytolytic. These events are accompanied by a significant increase in apoptosis and regeneration of hepatocytes as indicated in figure $(2 a-2 c)$ and $(3 a-3 c)$. the liver has a mark ability to regenerate. Resection of half of the human liver is followed within $2-4$ weeks by full restoration of the liver structure, size and function. The influx of the cytotoxic T lymphocytes will delay the accumulation of virus free hepatocyte. More so, chronically infected individuals can exhibit to some level $\mathrm{T}$ - cell accumulation, cytokine expression, and apoptosis. Our model predicted replacement of infected hepatocytes with regenerated cells which by known mechanism remain protected from reinfection in the individual after immune response.

\section{REFERENCES}

1. Alexander Rezounenko (20100. Viral infection model with diffusion and stateb dependent delay stability of classical solution. Mathematics subject classification: 93C23,34K20, 35K57, 97M60.

2. Burton T A. (1985). stability and periodic solutions of ordinary and functional differential equations. Academic Press, New York.

3. Cain D, Reynolds G (2006). The impact of facilities on recruitment and retention of students. APPA's centre for facilities research papers 2006.

4. Chinebu T. I, Aja R.O and Mbah G.C.E (2017) An intracellular Differential Equation Model for the Dynamics of Hepatitis B virus (Hbv) infection with immune control. International Journal of Precious Engineering Research and Application (IJPERA) 2(5), 1 - 11.

5. Ciupe S M,Ribeiro R M, Nelson P W, Perelson A $S$ (2007). "The role of cells refractory to productive infection in acute hepatitis B viral dynamics. Proceedings of the national academy of sciences of the united states of America, vol. 104,no.12,pp. 5055-5055.

6. Coffin C, Mulrooncy-Cousins P, Van Marle G, Roberts J, MichalaK J, Terrault N. (2011). Hepatitis $B$ virus (HBV) quesispecies in hepatic and extra hepatic viral reservoirs in liver transplants on prophylactic therapy. liver transpl 17(18): 955-62 doi: 1002/ it 22312 PMID 21462295.

7. Dayun $\mathrm{Wu}$, Yongmei Su (2014). Dynamical behaviour of a delay differential equation of Hepatitis $\mathrm{B}$ Virus. The $8^{\text {th }}$ International Conference on Systems Biology (ISB) 978-1-47997394-4/14/\$31.00 (C) 2014 IEEE. 
8. Gourley S A, Kuang Y,Nagy J D (2008). Dynamics of a delay differential equation model of hepatitis B virus infection. Journal of Biological Dynamics 2:2, 140-153.

9. Guang Huang, Yasuhiro Takeuchi and Wanbiao Ma (2010). Lyapunov Functionals for Delay Differential Equations Model of Viral infections. Society for Industrial and Applied Mathematics, Journal on Applied mathematics (SIAM J.Appl Math) 70(7), 2693 - 2708 (16 pages)

10. Halanay A. (1966). Differential equations: stability, oscillation, time lags. . New York: Academic press Inc.

11. Hassard BD, Kazarinoff ND and Wan YH. (1981). Theory and application of Hopf Bifurcation,. New York. Cambridge University Press.

12. Jinliang Wang, Xinxin Tian (2013). Global stability of a delay differential equation of
Hepatitis B virus infection with immune response. Electonic Journal of Differential Equations Vol 2013 No. 94 pp1 - 11 ISSN: 1072 - 6691.

13. Kaifa Wang, Wendi Wang and Shiping Song (2007). Dynamics of an HBV Model with diffusion and Delay. Journal of Theoretical Biology 253(2008) 36-44.

14. Long C, Qi H, Huang S. (2008). Mathematical modelling of cytotoxic Lymphocyte-mediated immune response to Hepatitis B virus infection. Journal of Biomedicine and Biotechnology , 9 pages doi: 10.1155/743690.

15. Mac Donald N (1970). Time lags in Biological Models. Springer-Verlag, Berlin.

16. Rotich R T, Lagat E R (2014). The bounds of time lag and chemotherapeutic efficacy in the control of HIV/AIDS. International Journal of mathematical research. 3(6): 63-81. 